\title{
O efeito do banho de imersão na duração do trabalho de parto*
}

\author{
THE EFFECT OF IMMERSION BATHS ON THE LENGTH OF CHILDBIRTH LABOR \\ EL EFECTO DEL BAÑO DE INMERSIÓN EN LA DURACIÓN DEL TRABAJO DE PARTO
}

\author{
Flora Maria Barbosa da Silva', Sonia Maria Junqueira Vasconcelos de Oliveira ${ }^{2}$
}

\section{RESUMO}

Estudo experimental, randomizado do tipo ensaio-clínico, teve como objetivo identificar a influência do banho de imersão na duração do primeiro período clínico do parto e na frequiência e duração das contrações uterinas. Os dados foram coletados em uma maternidade pública filantrópica da cidade de São Paulo que atende, em média, 1.100 partos mensais. A amostra constou de 108 parturientes, 54 no grupo controle e 54 no experimental, que fizeram uso do banho de imersão. Os resultados demonstraram que o banho de imersão não modificou a duração do trabalho de parto e a freqüência das contrações uterinas. No entanto, a duração das contrações foi estatisticamente menor no grupo experimental (41,9 versus $44,6 \mathrm{~min})$. Concluiu-se que o banho de imersão é uma alternativa para o conforto da mulher, durante o trabalho de parto, por oferecer alívio à parturiente, sem interferir na progressão do parto sem trazer prejuízos ao recém-nascido.

\section{DESCRITORES}

Parto.

Banhos.

Enfermagem obstétrica.

Contração uterina.

\section{ABSTRACT}

The objectives of this experimental, randomized, controlled trial study were to evaluate the effect of immersion baths on the length of the first stage of childbirth labor and on the frequency and length of the uterine contractions. Data were collected in a philanthropic public maternity hospital of the city of São Paulo whose month average is 1,100 births. The sample was comprised of 108 women in labor - 54 in the control group and 54 in the experimental group that had immersion baths. The results showed that immersion baths did not have any influence on the length of labor and on the frequency of uterine contractions. However, the length of contractions was statistically shorter in the experimental group (experimental 41.9 versus control $44.6 \mathrm{~min}$ ). The conclusion was that immersion baths are an alternative for the woman's comfort during labor, since it provides relief to her without interfering on the labor progression or jeopardizing the baby.

\section{KEY WORDS}

Parturition.

Baths.

Obstetrical nursing.

Uterine contraction.

\section{RESUMEN}

Este estudio experimental, randomizado del tipo ensayo-clínico, tuvo como objetivo identificar la influencia del baño de inmersión en la duración del primer período clínico del parto y en la frecuencia y duración de las contracciones uterinas. Los datos fueron recolectados en una maternidad pública filantrópica de la ciudad de São Paulo que atiende, en promedio, 1,100 partos mensuales. La muestra constó de 108 parturientas, 54 en el grupo control y 54 en el experimental, que hicieron uso del baño de inmersión. Los resultados demostraron que el baño de inmersión no modificó la duración del trabajo de parto y la frecuencia de las contracciones uterinas. Entre tanto, la duración de las contracciones fue estadísticamente menor en el grupo experimental (41,9 versus 44,6min). Se concluye que el baño de inmersión es una alternativa para el confort de la mujer, durante el trabajo de parto, por ofrecer alivio a la parturienta, sin interferir en la progresión del parto y ocasionar perjuicios al recién-nacido.

\section{DESCRIPTORES}

Parto.

Baños.

Enfermería obstétrica.

Contracción uterina.

\footnotetext{
* Extraído da Dissertação "O efeito do banho de imersão na dor e na duração do trabalho de parto", Escola de Enfermagem da Universidade de São Paulo (EEUSP), 2004.

1 Enfermeira Obstétrica da Casa de Parto do Itaim Paulista. Aluna do Programa de Pósgraduação da EEUSP, nível Mestrado. Membro do grupo de pesquisa: Enfermagem e assistência ao parto: modelos, agentes e práticas. do Departamento de Enfermagem Materno-Infantil e Psiquiátrica da EEUSP.

florambs@hotmail.com

2 Enfermeira Obstétrica. Professor Doutor do Departamento de Enfermagem Materno-Infantil e Psiquiátrica da EEUSP.

Coordenadora do grupo de pesquisa: Enfermagem e assistência ao parto: modelos, agentes e práticas. soniaju@usp.br
} 


\section{INTRODUÇÃO}

Uma das mais importantes tarefas dos prestadores de cuidados à mulher durante o trabalho de parto, é proporcionar condições de tolerância à dor e ao desconforto. Neste sentido, diversas estratégias têm sido desenvolvidas, tais com: o uso do óxido nitroso, opiáceos, alcalóides e, mais, recentemente, anestesias loco-regionais ${ }^{(1)}$.

Os inconvenientes do emprego de métodos farmacológicos de analgesia da parturiente, são apontados em vários estudos que ressaltam a interferência na progressão fisiológica do trabalho de parto e os efeitos adversos sobre o feto e a mãe, cujas repercussões a longo prazo ainda não estão bem definidas ${ }^{(2-6)}$.

Para a Organização Mundial de Saúde é essencial que métodos não-farmacológicos de alívio da dor sejam explorados, por serem mais seguros e acarretarem menos intervenções. Aí se incluem massagens, movimentação livre, exercícios respiratórios e a utilização de água em banhos de aspersão e imersão. Estas práticas são classificadas pela OMS como Categoria $\boldsymbol{C}$ - práticas em relação às quais não existem evidências suficientes para apoiar uma recomendação clara e que devem ser utilizadas com cautela até que mais pesquisas esclareçam a questão( ${ }^{(6)}$.

Diversos autores que avaliaram os efeitos do banho de imersão no trabalho de parto verificaram que esta intervenção pode influenciar o padrão das contrações uterinas e a duração do trabalho de parto. O pioneiro da descrição do uso do banho de imersão durante o trabalho de parto e parto foi Michel Odent ${ }^{(7)}$, no relato em que descreve a experiência no

Para a Organização Mundial de Saúde é essencial que métodos não-farmacológicos de alívio da dor sejam explorados, por serem mais seguros e acarretarem menos intervenções atendimento a 100 parturientes que deram à luz na água. Conforme relata o autor, nessas condições o trabalho de parto pode desenvolver-se de forma particularmente rápida, se bem que não tenha sido feita análise estatística para comprovar esta observação.

No Amparo Maternal, situado na zona sul de São Paulo, a assistência à parturiente durante o trabalho de parto e o parto normal vem sendo realizada em sua totalidade por enfermeiras obstétricas desde janeiro de 1999. Dentre as práticas realizadas pela equipe inclui-se o uso dos banhos de imersão, como forma de oferecer conforto à parturiente. Nesse sentido, surgiu a necessidade de avaliar a influência dessa prática no trabalho de parto.

\section{OBJETIVOS}

1 - Analisar a influência do banho de imersão na duração do primeiro período clínico do trabalho de parto;

2-Avaliar a influência do banho de imersão na freqüência e duração das contrações uterinas durante o trabalho de parto.

\section{CASUÍSTICA E MÉTODO}

Trata-se de um ensaio clínico experimental, controlado e randomizado sobre o uso do banho de imersão em nulíparas durante o trabalho de parto.

O estudo foi realizado no Centro de Parto Normal (CPN) do Amparo Maternal (AM), que é uma maternidade filantrópica, situada na zona sul da capital paulista. Esta instituição recebe gestantes e parturientes de toda a cidade de São Paulo, é vinculada ao Sistema Único de Saúde (SUS) e assiste em média 1.100 partos mensais, considerando-se o ano de 2002. A assistência aos partos normais é realizada em sua totalidade por enfermeiras obstétricas.

A população do estudo constituiu-se de mulheres que deram à luz por parto normal e foram assistidas por enfermeiras obstétricas no CPN-AM, e foi dividida em dois grupos: Grupo Controle - formado por parturientes que não fizeram uso do banho de imersão, como forma de alívio da dor durante o trabalho de parto e Grupo Experimental - composto por parturientes que fizeram uso do banho de imersão como método de alívio da dor.

No estudo foram incluídas as parturientes que atenderam aos seguintes critérios: ser nulípara, ou seja, não ter parto anterior; estar com gestação a termo e feto em apresentação cefálica fletida; não ser portadora de intercorrências clínicas ou obstétricas; apresentar cervicodilatação igual ou inferior a 6 centímetros no momento da admissão hospitalar; bolsa das águas íntegra ou rota espontaneamente em período inferior a 6 horas antes da admissão e estar com dinâmica uterina de duas ou mais contrações em dez minutos.

Foram excluídas as mulheres que apresentaram inter-corrências clínicas ou obstétricas no decorrer do trabalho de parto que justificassem a realização de parto cesariana e utilização de medicação que não seja a ocitocina endovenosa.

A amostra foi composta por 108 mulheres, sendo 54 no Grupo Controle e 54 no Grupo Experimental. A randomização foi feita por uma tabela, aplicada no momento de abordagem da parturiente e sua inclusão no estudo. As mulheres excluídas foram substituídas automaticamente por outras, seguindo a mesma tabela.

Para o cálculo do tamanho da amostra, utilizou-se o estu$\mathrm{do}^{(8)}$ realizado no CPN-AM, considerando o total de partos assistidos por enfermeiras obstétricas no período de dois meses, entre 1/7/01 a 31/8/01.

Assumindo um erro de 5\% e dois desvios padrão, tem-se que $n=108$, portanto, cada grupo foi composto por 54 pacientes. 
Os dados foram coletados pela própria pesquisadora, nas seguintes etapas, realizadas sucessivamente:

Abordagem e randomização - No primeiro contato com a parturiente, foi explicada a finalidade do estudo e soli-citada a assinatura do termo de consentimento livre e esclarecido.

1. ${ }^{a}$ avaliação - Feita logo após a abordagem, avaliando-se a dinâmica uterina nas parturientes dos grupos Controle e Experimental.

2. ${ }^{a}$ avaliação - As parturientes de ambos os grupos foram avaliadas quanto à dinâmica uterina, temperatura corporal e do ambiente (pré-parto) ao apresentarem cervicodilatação de 6 a 7 centímetros. As mulheres dos dois grupos, que foram admitidas no estudo com cervicodilatação de 6 centímetros, tiveram o intervalo de uma hora entre $1 .{ }^{\mathrm{a}} \mathrm{e} 2 .^{\mathrm{a}}$ avaliações.

Procedimento do banho de imersão - As parturientes do Grupo Experimental foram encaminhadas ao banho de imersão, durante o período de 40 a 60 minutos. A mulher pôde optar pela temperatura da água, conforme sua preferência e a seguir esta temperatura foi mensurada.

3. ${ }^{a}$ avaliação - Logo após o banho, foi feita nova avaliação da dinâmica uterina no Grupo Experimental. O Grupo Controle foi submetido à 3 . $^{\mathrm{a}}$ avaliação da dinâmica uterina uma hora após a $2 \cdot{ }^{\text {a }}$ avaliação.

Os grupos Controle e Experimental tiveram entre a 2. ${ }^{\mathrm{a}} \mathrm{e}$ $3{ }^{a}$ avaliações o intervalo de uma hora.

$\mathrm{Na}$ administração de ocitocina, foram usadas 5UI em $500 \mathrm{ml}$ de soro glicosado a $5 \%$ ou realizada a amniotomia, quando a cervicodilatação manteve-se inalterada por mais de duas horas.
O tempo de duração do trabalho de parto foi calculado empregando-se como início o momento, em que foi detectada a cervicodilatação de 6 centímetros, até a dilatação total da cérvix uterina.

Os dados foram armazenados em um banco de dados do aplicativo Excel e a análise estatística foi realizada no programa SPSS versão 8.0. Inicialmente, foi feita uma análise descritiva dos dados, que se encontram em forma de tabelas ou gráficos. Para as variáveis quantitativas, foram usados valores das médias e medianas para resumir as informações, e valores dos desvios padrão, mínimo e máximo para indicar a variabilidade dos dados.

Para analisar as variáveis qualitativas, foram utilizados testes Qui-Quadrado de Pearson, exato de Fisher, t-Student e de Mann-Whitney, sendo considerados estatisticamente significantes os resultados, cujos níveis descritivos (valores de $\mathrm{p}$ ) foram inferiores a 0,05 .

\section{RESULTADOS}

Os grupos não apresentaram diferenças quanto à raça, idade materna, idade gestacional, gestação, preparo para o parto, estado marital, escolaridade, modo de ruptura da bolsa, coloração do líquido amniótico, uso de ocitocina, condição perineal, tempo de bolsa rota, dilatação ao usar a ocitocina, duração do trabalho de parto, duração do período expulsivo, temperatura do ambiente, temperatura da parturiente, peso do recém-nascido, Apgar do $1 .^{\circ}$ e $5{ }^{\circ}$ minuto.

\section{Dados relativos à duração do trabalho de parto}

Neste estudo, a duração do trabalho de parto foi considerada do momento em que se constatou cervicodilatação de seis centímetros, com a parturiente internada na maternidade campo de estudo, até a dilatação de dez centímentros, registrados em minutos.

Tabela 1 - Valores da média, desvio padrão, mediana, mínimo emáximo da duração do trabal ho de parto, em minutos, segundo os grupos - São Paulo, 2002-2003

\begin{tabular}{cccccccc}
\hline Grupo & $\mathrm{n}$ & Média & $\begin{array}{c}\text { Desvio } \\
\text { padrão }\end{array}$ & Mediana & Mínimo & Máximo & p-valor \\
\hline Controle & 54 & 260,4 & 135,7 & 230,0 & 40,0 & 620,0 & 0,885 \\
Experimental & 54 & 250,9 & 128,9 & 240,0 & 60,0 & 600,0 & \\
\hline
\end{tabular}

Teste Mann-Whitney

Nos resultados da Tabela 1, observa-se que os valores da média, desvio padrão e máximo da duração do trabalho de parto foram maiores para as mulheres do grupo con- trole do que para as do grupo experimental, embora sem diferença estatisticamente significativa entre eles. 
Tabela 2 - Valores da média, desvio padrão, mediana, mínimo e máximo da dilatação cervical na primeira, segunda eterceira avaliações, segundo os grupos - São Paulo, 2002-2003

\begin{tabular}{|c|c|c|c|c|c|c|c|c|}
\hline Avaliação & Grupo & $N$ & Média & $\begin{array}{l}\text { Desvio } \\
\text { padrão }\end{array}$ & Mediana & Mínimo & Máximo & $p$-valor \\
\hline \multirow{2}{*}{$1 .^{a}$} & Controle & 54 & 5,0 & 0,9 & 5,0 & 3,0 & 6,0 & \multirow{2}{*}{0,490} \\
\hline & Experimental & 54 & 5,1 & 0,9 & 5,0 & 3,0 & 6,0 & \\
\hline \multirow{2}{*}{$2 .^{a}$} & Controle & 54 & 6,3 & 0,5 & 0,5 & 6,0 & 7,0 & \multirow{2}{*}{0,409} \\
\hline & Experimental & 54 & 6,3 & 0,4 & 0,4 & 6,0 & 7,0 & \\
\hline \multirow{2}{*}{ 3. ${ }^{a}$} & Controle & 54 & 7,2 & 0,9 & 0,9 & 6,0 & 9,0 & \multirow{2}{*}{0197} \\
\hline & Experimental & 54 & 7,4 & 0,9 & 0,9 & 6,0 & 10,0 & \\
\hline
\end{tabular}

Teste Mann-Whitney

Quando realizada a comparação de cervicodilatação entre os grupos, verificou-se que não houve diferença estatisticamente significativa nas três avaliações.

\section{Dados relativos à freqüiência das contrações uterinas}

A freqüência das contrações foi calculada com a realização da dinâmica uterina, verificando-se o número de contrações em dez minutos (Tabela 3).

Tabela 3 - Valores da média, desvio padrão, mediana, mínimo e máximo da freqüência das contrações uterinas na primeira, segunda eterceira avaliações, segundo os grupos - São Paulo, 2002-2003

\begin{tabular}{|c|c|c|c|c|c|c|c|c|}
\hline Avaliação & Grupo & $N$ & Média & $\begin{array}{l}\text { Desvio } \\
\text { padrão }\end{array}$ & Mediana & Mínimo & Máximo & p-valor \\
\hline \multirow{2}{*}{$1 .^{a}$} & Controle & 54 & 2,9 & 0,8 & 3,0 & 1,0 & 4,0 & \multirow[b]{2}{*}{0,959} \\
\hline & Experimental & 54 & 2,9 & 0,9 & 3,0 & 1,0 & 4,0 & \\
\hline \multirow{2}{*}{$2 .^{a}$} & Controle & 54 & 3,2 & 0,9 & 3,0 & 1,0 & 6,0 & \multirow[b]{2}{*}{0,733} \\
\hline & Experimental & 54 & 3,2 & 0,8 & 3,0 & 2,0 & 5,0 & \\
\hline \multirow{2}{*}{ 3. ${ }^{a}$} & Controle & 54 & 3,5 & 0,9 & 3,0 & 1,0 & 5,0 & \multirow[t]{2}{*}{0,401} \\
\hline & Experimental & 54 & 3,4 & 0,8 & 3,0 & 1,0 & 5,0 & \\
\hline
\end{tabular}

Teste Mann-Whitney

Nos resultados da Tabela 3, nota-se que os valores das médias da frequiência das contrações uterinas entre os grupos foram semelhantes nas três avaliações, sem diferença estatisticamente significativa $(\mathrm{p}>0,05)$. Observa-se que houve um aumento destes valores ao longo das três avaliações, conforme o esperado na evolução do trabalho de parto fisiológico.

\section{Dados relativos à duração das contrações uterinas}

Os resultados apontados na Tabela 4 indicam a duração das contrações uterinas.

Tabela 4 - Val ores da média, desvio padrão, mediana, mínimo e máximo da duração das contrações uterinas (em segundos) na primeira, segunda eterceira avaliações, segundo os grupos - São Paulo, 2002-2003

\begin{tabular}{|c|c|c|c|c|c|c|c|c|}
\hline Avaliação & Grupo & $N$ & Média & $\begin{array}{l}\text { Desvio } \\
\text { padrão }\end{array}$ & Mediana & Mínimo & Máximo & $p$-valor \\
\hline \multirow{2}{*}{$1 .^{a}$} & Controle & 54 & 39,3 & 7,3 & 40,0 & 25,0 & 55,0 & \multirow[b]{2}{*}{0,335} \\
\hline & Experimental & 54 & 38,1 & 6,1 & 37,9 & 25,0 & 52,5 & \\
\hline \multirow{2}{*}{$2 .^{a}$} & Controle & 54 & 43,6 & 7,2 & 43,3 & 30,0 & 60,0 & \multirow[b]{2}{*}{0,257} \\
\hline & Experimental & 54 & 42,1 & 6,4 & 41,2 & 31,7 & 60,0 & \\
\hline \multirow{2}{*}{$3 .^{a}$} & Controle & 54 & 44,6 & 6,3 & 44,4 & 32,5 & 65,0 & \multirow[t]{2}{*}{ 0,030* } \\
\hline & Experimental & 54 & 41,9 & 6,5 & 40,0 & 30,0 & 60,0 & \\
\hline
\end{tabular}

Teste t-Student *estatisticamente significativo 
Na Tabela 4 verifica-se que na terceira avaliação houve diferença estatisticamente significativa entre os valores da duração da contração uterina entre os grupos $(\mathrm{p}=0,030)$, sendo o valor da média do grupo experimental menor do que o valor da média no grupo controle (41,9 versus 44,6 segundos).

\section{DISCUSSÃO}

O processo fisiológico na gravidez que culmina no início do trabalho de parto ainda não está esclarecido. É sabido que, em geral, o miométrio não apresenta atividade contrátil que modifique o colo uterino, até aproximadamente, a trigésima sexta semana de gravidez. Após esse período prolongado de repouso, há necessidade de uma fase caracterizada por contrações uterinas ritmadas que produzam dilatação da cérvice.

Nesse sentido, a referência do início dessas contrações deve ser obtida da própria parturiente, estando, portanto, sujeita a $\operatorname{erros}^{(9)}$. Segundo esses autores, a única avaliação objetiva que se tem da evolução do trabalho de parto, é a mensuração da cervicodilatação uterina, pelo toque vaginal. Deste modo, neste estudo, optou-se por mensurar a duração do trabalho de parto do momento em que foi constatada a cervicodilatação de seis centímetros, estando a parturiente admitida no centro de parto normal, campo do estudo, até o momento em que se constatou a dilatação total.

Alguns pesquisadores aventaram a hipótese de o uso do banho de imersão poder abreviar o trabalho de parto. No estudo ${ }^{(10)}$ realizado com 160 parturientes, o grupo controle foi constituído por 72 mulheres e o experimental por 88 mulheres. A cervicodilatação era de 5 centímetros no início do estudo, para ambos os grupos. Verificou-se uma velocidade significativamente maior de dilatação cervical entre as mulheres que fizeram uso de banhos mornos em banheiras $(2,5 \mathrm{~cm} /$ hora) quando comparadas com aquelas que não utilizaram banho (1,2 cm/hora), sem que houvesse diferença estatística na duração total do trabalho de parto. Merece comentar que esses autores consideraram como tempo total do trabalho de parto aquele decorrido entre o início das primeiras contrações regulares e a expulsão fetal. Alguns fatores podem ter influência, como a variedade de posição, o peso do feto, os esforços expulsivos e a posição adotada pela parturiente, entre outros.

Também não foram encontradas diferenças na duração do trabalho de parto em um estudo randomizado ${ }^{(11)}$, no qual avaliaram 93 mulheres, destas 45 fizeram uso do banho e 48 não o realizaram. Ambos os grupos fizeram uso de todos os métodos disponíveis para alívio da dor. As mulheres do gru- po experimental permaneceram na banheira pelo período de 30 a 45 minutos. Não houve diferença na duração do trabalho de parto ou na progressão da dilatação cervical entre os grupos. Os resultados corroboram os achados da pesqui$\mathrm{sa}^{(12)}$, que também não verificou diferenças nestas variáveis, em um estudo com 110 nulíparas (56 no grupo controle, 54 no experimental). Os autores dos dois trabalhos não descreveram como foi feita a mensuração da duração do trabalho de parto.

Da mesma forma, investigação ${ }^{(13)}$ realizada comparando as mulheres que utilizaram o banho de imersão com as que não o fizeram, constataram que a duração do trabalho de parto também não apresentou diferença estatisticamente significativa.

No atual estudo, não foi encontrada diferença estatisticamente significativa, com relação à duração do trabalho de parto em minutos, entre os grupos controle e experimental (Tabela 1), embora o grupo experimental tenha apresentado média inferior à do grupo controle (250,9 versus $260,4 \mathrm{~min})$.

O primeiro estágio do trabalho de parto, conhecido como período de esvaecimento e dilatação cervical, começa quando as contrações uterinas têm freqüência, intensidade e duração suficientes para modificarem a cérvix uterina. $\mathrm{O}$ intervalo entre as contrações diminui de forma gradativa; a princípio dura cerca de 10 minutos, no início do primeiro estágio do trabalho de parto, chegando a apenas um minuto e meio no segundo estágio. $\mathrm{Na}$ fase ativa do trabalho de parto, a duração de cada contração varia de 30 a 90 segundos, sendo em média de um minuto ${ }^{(9)}$. Não há demarcação nítida entre o trabalho de parto e o parto, mas uma transição gradual, o que dificulta estabelecer o início da dilatação. Assim, no período de dilatação as contrações uterinas são, inicialmente, em número de duas a três em 10 minutos, chegando a atingir cinco em 10 minutos ao fim do período ${ }^{(14)}$.

As contrações uterinas são dolorosas por causas não totalmente estabelecidas, sendo aventadas algumas hipóteses: hipoxia do miométrio contraído, compressão de gânglios nervosos na cérvix e na parte inferior do útero pelos feixes musculares entrelaçados, estiramento cervical durante a dilatação e estiramento do peritônio subjacente ao fundo uterino ${ }^{(9)}$. Dentre as alterações fisiológicas, que acompanham a dor durante o trabalho de parto, destacam-se o aumento do consumo de oxigênio cerca de $40 \%$ acima dos níveis anteriores ao trabalho de parto, levando à alcalose respiratória que pode ocasionar hipoxemia fetal; aumento progressivo do débito cardíaco materno; aumento dos níveis de adrenalina, noradrenalina, cortisol e ACTH no sangue materno e acidose metabólica materna $\operatorname{progressiva}^{(15)}$. 
Assim, observa-se a necessidade de aliviar a dor e a ansiedade da parturiente, uma vez que estes mecanismos podem prejudicar o feto e afetar a progressão fisiológica do trabalho de parto.

Alguns autores alegam a possibilidade de o banho diminuir as contrações uterinas. Investigação ${ }^{(16)}$ que comparou o uso do banho de imersão em fase precoce (dilatação cervical inferior a $5 \mathrm{~cm}$ ) e em fase tardia do trabalho de parto (dilatação cervical superior a $5 \mathrm{~cm}$ ), constatou que o grupo que fez uso do banho precocemente teve maior necessidade de infusão de ocitocina e de analgesia peridural, provavelmente por ter um trabalho de parto mais prolongado $(9,8$ versus 8,5 horas) que o grupo do banho realizado tardiamente.

Não foi observado que, durante o trabalho de parto, o uso do banho de imersão não influenciou o padrão de contra-tilidade uterina. $\mathrm{O}$ aumento da freqüência das contrações foi similar nos grupos controle e experimental, no decorrer do trabalho de parto $^{(11)}$.

No presente estudo, não foi verificada diferença estatisticamente significativa entre os valores das médias da frequiência das contrações uterinas entre os grupos controle e experimental nas três avaliações. Por outro lado, a média da duração das contrações foi menor no grupo experimental que no grupo controle, com diferença estatística (41,9 contra 44,6 segundos), conforme mostra a Tabela 4. Verificou-se esta diferença na terceira avaliação, após o grupo experimental ter realizado o banho de imersão.

\section{CONCLUSÕES}

Este trabalho procurou explorar de forma científica o efeito de uma prática de conforto do trabalho de parto que vem sendo utilizada em muitos países. Os altos níveis de intervenção adotados, muitas vezes, de maneira inoportuna não proporcionam melhora nos resultados obstétricos, porém encarecem a assistência e têm alto potencial iatrogênico, além de diminuirem a satisfação da mulher com o evento do nascimento e parto. $\mathrm{O}$ banho de imersão permite que a mu-

\section{REFERÊNCIAS}

(1) Findley I, Chamberlain G. ABC of labour care. Relief of pain. BMJ. 1999;318(7188):927-30.

(2) Balaskas J. Parto ativo: guia prático para o parto natural. São Paulo: Ground; 1993.

(3) Chua S, Arulkumaran S. Intrapartum care. Aust N Z J Obstet Gynaecol. 1997;37(1):25-35.

(4) Lieberman E. No free lunch on labor day. The risks and benefits of epidural analgesia during labor. J Nurse Midwifery. 1999;44(4):394-8. lher assuma o comando da situação, pois ela pode mobilizar seus recursos para buscar o bem-estar durante o processo do nascimento.

O uso de métodos não-farmacológicos, como o banho de imersão no alívio da dor durante o trabalho de parto tem as vantagens de reduzir e postergar o uso de fármacos no controle da dor, proporcionando condições para a colaboração ativa da parturiente e permitindo maior participação do acompanhante.

Observa-se que há dificuldade em comparar os resultados do presente trabalho com os demais estudos, visto que a maioria não descreve como foi feita a mensuração da duração do trabalho de parto ou quanto tempo a mulher permaneceu no banho de imersão e em que período do trabalho de parto. Além disso, muitos estudos incluem nulíparas e multíparas na amostra, o que pode comprometer a média da duração do trabalho de parto, uma vez que a duração do trabalho de parto na multípara tende a ser mais curta.

Assim, procurou-se observar uma metodologia que fornecesse confiança nos dados obtidos e subsídios aos profissionais que queiram fazer uso dessa prática.

Neste estudo, não foram encontrados resultados negativos do uso do banho de imersão na vitalidade do recém nascido, visto que os índices de Apgar mostraram-se satisfatórios, tanto no grupo controle como no experimental. Vale mencionar que seria interessante observar os índices de infecção puerperal entre as mulheres do estudo. No entanto, isto não foi possível em razão de o local da pesquisa não possuir um serviço de retorno das puérperas. Estas são provenientes de toda a cidade de São Paulo, procuram atendimento em serviços próximos às suas casas, quando apresentam alguma intercorrência no período puerperal, o que dificulta o rastreamento dos casos de infecção.

O presente estudo constatou que o banho de imersão é uma opção viável para o conforto da parturiente, sem interferir na progressão do trabalho de parto.

(5) Nyberg K, Buka SL, Lipsitt LP. Perinatal medication as a potential risk factor for adult drug abuse in a North American Cohort. Epidemiology. 2000;11(6):715-6.

(6) Organização Mundial de Saúde (OMS). Assistência ao parto normal: um guia prático. Brasília: OPAS/USAID; 1996. (OMS/ SRF/MSM/96.24).

(7) Odent M. Birth under water. Lancet. 1983;2(8365/66):1476-7. 
(8) Silva FMB, Oliveira SMJV, Koiffman MD, Osava RH. Caracterização de parturientes atendidas em um centro de parto normal. In: Anais do $2^{\circ}$ Encontro Internacional de Pesquisa em Enfermagem: Trajetória Espaço-Temporal da Pesquisa; 2002 out. 28-31; Águas de Lindóia (SP). [CD-ROM]. São Paulo: EEUSP; 2002.

(9) Cunningham FG, MacDonald PC, Gant NF, Leveno KJ, Giltrasp III LC, Hankins GDV et al. Williams obstetrícia. $20^{a}$ ed. Rio de Janeiro: Guanabara Koogan; 2000. Trabalho de parto e parto normais; p. 225-74.

(10) Lenstrup C, Schantz A, Berget A, Feder E, Rosen $\varnothing$ H, Hertel $\mathrm{H}$. Warm tub bath during delivery. Acta Obstet Gynecol Scand. 1987;66(8):709-12.

(11) Schorn MN, McAllister JL, Blanco JD. Water immersion and the effect on labor. J Nurse Midwifery. 1993;38(6):336-42.

(12) Cammu H, Clasen K, Van Wettere L, Derde MP. "To bathe or not to bath" during the first stage of labor. Acta Obstet Gynecol Scand. 1994;73(6):468-72.
(13) Robertson PA, Huang LJ, Croughan-Minihane MS, Kilpatrick SJ. Is there an association between water baths during labor and the development of chorioamnionitis or endometritis? Am J Obstet Gynecol. 1998;178(6):1215-21.

(14) Rezende, J. Obstetrícia básica. 9a ed. Rio de Janeiro: Guanabara Koogan; 2002. O parto: estudo clínico e assistência; p. $328-45$.

(15) Brasil. Ministério da Saúde. Secretaria de Políticas de Saúde. Área Técnica de Saúde da Mulher. Parto, aborto e puerpério: assistência humanizada à mulher. Brasília; 2001.

(16) Eriksson M, Mattsson L-A, Ladfors L. Early or late bath during the first stage of labour: a randomized study of 200 women. Midwifery. 1997;13(3):146-8. 